\title{
Rapid Reduction of Syrinx Associated With Traumatic Intracranial Hypotension by Direct Surgery
}

\section{-Case Report-}

\author{
Tatsuya OHTONARI, Nobuharu NishiHARA, Taisei OTA, Satoshi KOBANAWA*, \\ Shinzo OTA*, Wataru DANJO**, and Tsunemaro KOYAMA*** \\ Departments of Spinal Surgery and \\ *Neurosurgery, Brain Attack Center Ota Memorial Hospital, Fukuyama, Hiroshima; \\ **Department of Emergency Medicine, Okayama University Graduate School of \\ Medicine, Dentistry and Pharmaceutical Sciences, Okayama; and \\ ${ }^{* * *}$ Clinical Institute for Spinal Disorders, Otsu, Shiga
}

\begin{abstract}
A woman in her early twenties presented with cerebellar tonsillar herniation with syrinx in the cervicothoracic spinal cord manifesting as postural headache after suffering trauma to the hip. She was treated under a diagnosis of traumatic intracranial hypotension. Cranial magnetic resonance (MR) imaging demonstrated tonsillar herniation to the upper rim of the C1 lamina, associated with effacement of the basal cisterns and flattening of the pons against the clivus, sagging of the optic chiasm, and protrusion of the pituitary gland into the suprasellar cistern. Spinal MR imaging and computed tomography (CT) myelography showed cervicothoracic syrinx and arachnoid diverticulum. Three applications of epidural blood patches produced no improvement. Surgery identified cerebrospinal fluid leakage from two small holes in the spinal dural sac, slightly proximal from the origin of the left L1 root sleeve, and arachnoid diverticulum. These two holes were sutured and a few sheets of gelatin sponge were placed around the arachnoid diverticulum and sealed with fibrin glue. The cervicothoracic syrinx was reduced significantly and tonsillar herniation disappeared within 10 days. Simple surgical repair of the dural tears may rapidly improve symptoms and imaging findings in patients with tonsillar herniation caused by traumatic intracranial hypotension.
\end{abstract}

Key words: intracranial hypotension, syringomyelia, epidural blood patch, surgery

\section{Introduction}

Spontaneous intracranial hypotension is a syndrome of reduced intracranial volume that results from cerebrospinal fluid (CSF) leakage from the spinal subarachnoid space. ${ }^{12,16)}$ Traumatic intracranial hypotension is a variant triggered by body impact. Most cases of spontaneous intracranial hypotension are thought to resolve spontaneously or improve with simple treatment with an intravenous drip, whereas traumatic intracranial hypotension rarely resolves spontaneously. Chronic traumatic intracranial hypotension causes sagging of the intracranial structures, most prominently in the posterior fossa. ${ }^{1)}$ Generally, patients present with headache that worsen in the upright posture but

Received February 25, 2008; Accepted September 10, 2008 are relieved by lying down. ${ }^{1,3,9)}$ Occasionally, a chronic disease process may obscure the postural headache. ${ }^{1,7)}$ Other symptoms are related to stretching or displacement of the intracranial structures, and include ataxia, visual deficits, diplopia, tinnitus, vertigo, and hearing disturbances. ${ }^{3)}$ Symptoms related to the spine are seldom seen, and limited mostly to neck pain and radiculopathy, although cervical epidural CSF may have led to myelopathy in one case. ${ }^{3,6,11)}$ Conservative treatment for spinal CSF leakage usually consists of rest or intravenous drip. Epidural blood patch (EBP) can be applied, but the effectiveness is about $70 \%$, even if the procedure is performed repeatedly. Therefore, direct surgery is indicated if such indirect treatment proves unsuccessful. ${ }^{2,10)}$

We report a case of intracranial hypotension with cervicothoracic syrinx secondary to mild trauma treated by surgical repair after three applications of 

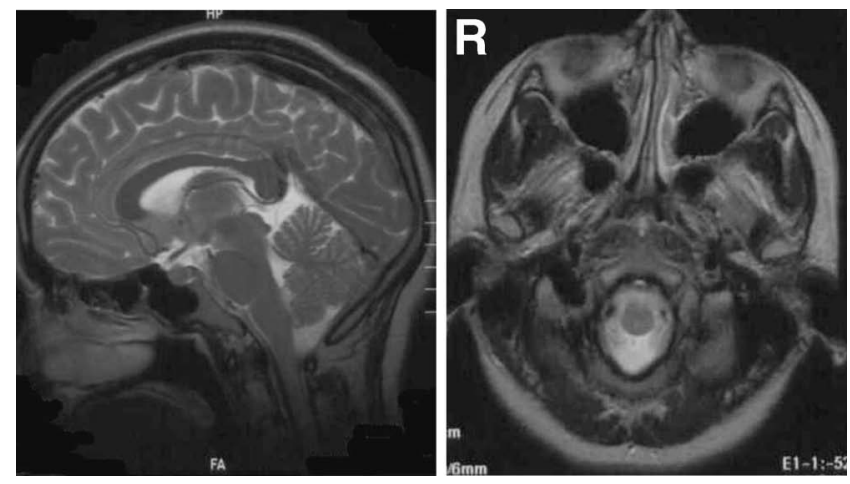

Fig. 1 Intracranial sagittal (left) and axial (right) $\mathrm{T}_{2}$-weighted magnetic resonance images obtained at a local clinic before admission showing no apparent intracranial abnormality.

EBP were unsuccessful.

\section{Case Report}

A woman in her early twenties suffered chronic headache after she had tripped and hit her hip against a stair. The headache was aggravated by sitting or standing but relieved by lying down. She consulted a local clinic and underwent magnetic resonance (MR) imaging, but no intracranial abnormality was detected (Fig. 1). She tolerated her headache with the help of analgesics for 2.5 years before coming to our hospital.

Multiplanar cranial MR imaging was performed using a 1.5-tesla scanner, with and without intravenous administration of contrast agents. The MR images revealed tonsillar herniation to the upper rim of the C1 lamina, associated with effacement of the basal cisterns and flattening of the pons against the clivus. Furthermore, sagging of the optic chiasm was present with protrusion of the pituitary gland into the suprasellar cistern (Fig. 2). No diffuse enhancement of the intracranial dura mater was detected.

Cranial imaging was followed by MR imaging of the spine. MR imaging from the subocciput to upper thoracic spine confirmed the presence of a syrinx in the cervicothoracic spinal cord from the upper rim of the $\mathrm{C} 7$ vertebral body to the lower rim of the $\mathrm{T} 2$ vertebral body with the pre-syrinx state in the rostral cervical spinal cord (Fig. 3A). MR imaging of the lumbar spine showed fluid collection in the left multifidus muscle from the L1 to T12 levels (Fig. 3B, C). On the other hand, MR myelography revealed no apparent point of CSF leakage. Computed tomography (CT) myelography indicated the presence of an

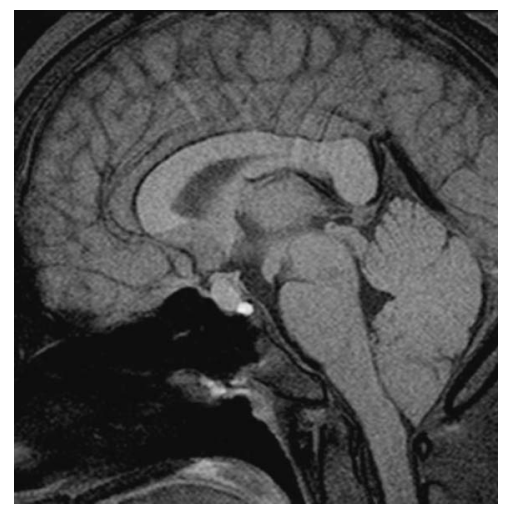

Fig. 2 Sagittal $T_{1}$-weighted magnetic resonance image of the brain revealing tonsillar herniation to the upper rim of the C1 lamina, associated with effacement of the basal cisterns and flattening of the pons against the clivus, and sagging of the optic chiasm with protrusion of the pituitary gland into the suprasellar cistern.
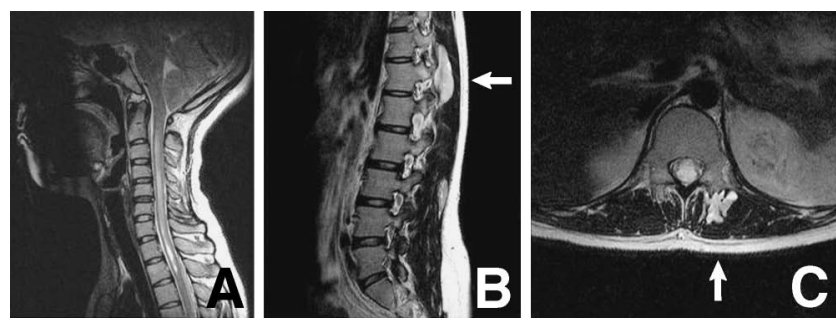

Fig. 3 A: Sagittal $T_{2}$-weighted magnetic resonance (MR) image from the foramen magnum to the upper thoracic region showing a syrinx in the cervicothoracic spinal cord from the upper rim of the $C 7$ vertebral body to the lower rim of the T2 vertebral body with the pre-syrinx state in the rostral cervical spinal cord. B, C: Sagittal (B) and axial (C) $\mathrm{T}_{2^{-}}$ weighted $M R$ images of the lumbar spine showing fluid collection in the left multifidus muscle from the L1 to T12 levels (arrows).

arachnoid diverticulum at the axilla of the left L1 root sleeve (Fig. 4).

Our recognition of this case was cerebellar tonsillar herniation with cervicothoracic syrinx caused by traumatic intracranial hypotension and CSF leakage at the lumbar spine. EBP was applied three times, but none of her symptoms or MR imaging findings showed any improvement. Therefore, direct surgical suture of the spinal dural tears and additional repair with Gelfoam ${ }^{\mathbb{R}}$ (Pharmacia \& Upjohn, New York, N.Y., U.S.A.) and fibrin glue at the axilla of the left 


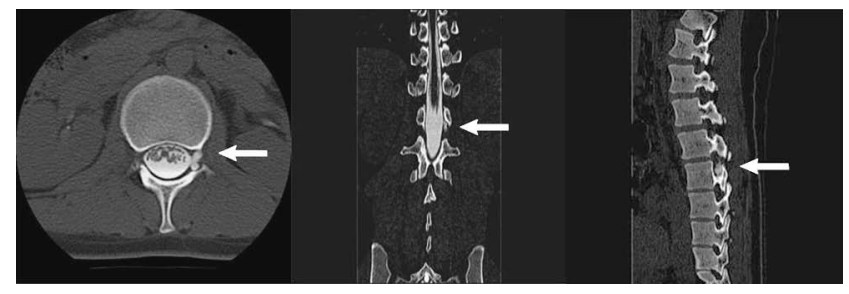

Fig. 4 Computed tomography myelograms indicating the presence of an arachnoid diverticulum at the axilla of the left $L 1$ root sleeve (arrows).

L1 root sleeve was planned. After the laminectomy of the L1 lamina, CSF containing venous blood began to flow out as soon as the yellow ligament was excised. Partial severe adhesion to the spinal dura mater by granulation was confirmed, which was probably affected by the EBP. After this adhesion was dissected, an arachnoid diverticulum was confirmed at the origin of the left L1 root sleeve (Fig. 5). However, CSF leakage was found, not from this diverticulum, but from two pinholes in the dorsal thin dural sac at the origin of the root sleeve. These holes were sutured with 8-0 nylon stitches (Fig. 6). After the CSF leakage from these dural tears was controlled, a few sheets of Gelfoam ${ }^{\circledR}$ were placed around the arachnoid diverticulum and sealed with fibrin glue because of the possibility of invisible tears in the ventral dural sac.

The patient spent 5 days recovering in bed, but noted gradual relief of her headache on the 6th day after the surgery, and was free from headache on the 9th day even if she stood for a long time. MR imaging of the head on the 10th day showed that the tonsils had ascended and returned to their normal shape. The basal cistern was well visualized, the pons had a normal shape, and the pontomedullary sulcus was well visualized. The upward bulging of the pituitary was no longer present and the compression of the chiasm had resolved (Fig. 7A). These findings indicated resolution of tonsillar herniation and intracranial hypotension. MR imaging of the cervical spine on the 10th day demonstrated almost complete resolution of the pre-syrinx state and considerable reduction of the syrinx (Fig. 7B). MR imaging of the thoracic spine demonstrated a residual syrinx.

Her positional headache has not reappeared at all since the operation. Repeat MR imaging has demonstrated no recurrence of tonsillar herniation or syrinx in the cervical spinal cord, and the syrinx in the thoracic spinal cord is showing a tendency to reduce.

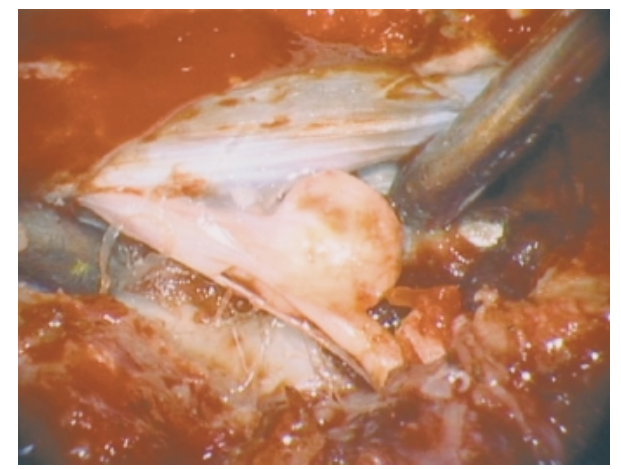

Fig. 5 Intraoperative photograph confirming the arachnoid diverticulum at the origin of the left $L 1$ root sleeve.

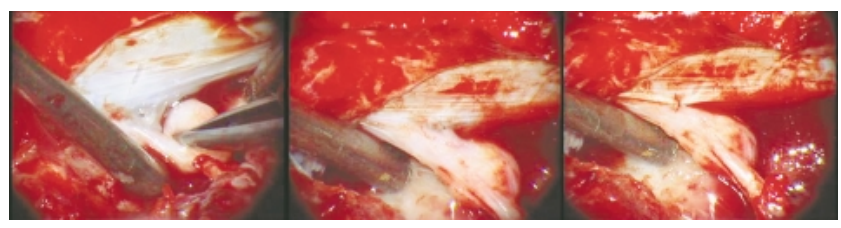

Fig. 6 Intraoperative photographs showing cerebrospinal fluid leakage from two pinholes in the dorsal dural sac at the origin of the root sleeve. Both holes were sutured with 8-0 nylon stitches.
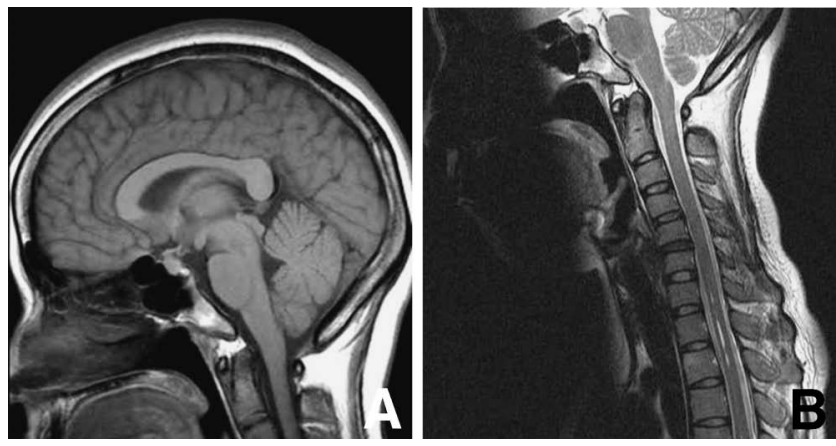

Fig. 7 A: Postoperative sagittal $\mathrm{T}_{1}$-weighted magnetic resonance (MR) image of the head on the 10th day after surgery showing the tonsils had ascended and returned to their normal shape. The upward bulging of the pituitary was no longer present and the compression of the chiasm had resolved. The basal cistern was well visualized, the pons had a normal shape, and the pontomedullary sulcus was well visualized. $B$ : Sagittal $T_{2^{-}}$ weighted MR image of the cervical spine on the 10th day demonstrating almost complete resolution of the pre-syrinx state and considerable reduction of the syrinx. 


\section{Discussion}

Intracranial MR imaging of patients with spontaneous intracranial hypotension often demonstrates findings of intracranial hypotension, tonsillar herniation, descending transtentorial herniation, flattering of the pons against the clivus, obliteration of intracranial cisterns, descent of the canaliculus, sagging of the optic chiasm, and protrusion of the pituitary gland through the diaphragma sellae into the suprasellar cistern. ${ }^{1,4,9)}$ Tonsillar herniation is typically identified as associated with Chiari I malformation, but other findings of brain sagging or a history of postural headache indicate the possibility of intracranial hypotension. In fact, previous patients presenting with headache with diagnoses of Chiari I malformation were actually suffering from spontaneous intracranial hypotension. ${ }^{1)}$ Intracranial diffuse enhancement of the dura mater is another common finding. ${ }^{1,3,4,9)}$ However, meningeal enhancement was absent in a patient with intracranial hypotension. ${ }^{14)}$ Our case also revealed no meningeal enhancement. Moreover, diffuse dural enhancement may not be specific to intracranial hypotension but may also be involved in various conditions such as infection, neoplasm, surgical invasive response, subarachnoid hemorrhage, and idiopathic diseases.

Intracranial hypotension is thought to be caused by CSF leakage from the spine. Spinal MR imaging findings include the presence of epidural fluid collection, pachymeningeal enhancement, and dilated epidural veins. ${ }^{3,6,8,11,13)}$ Communications between such extradural fluid collections and the subarachnoid space are known, indicating that these collections represent extravasated CSF.4,11,12,16) Radionuclide cisternography or CT myelography are performed to locate the site of the leak. In our case, CT myelography easily located the site of the leak, facilitated by previous detection of the CSF collection in the multifidus muscle.

Some syringes associated with intracranial hypotension result from drainage of the CSF from the lumbar subarachnoid space. Seven patients developed tonsillar herniation and syringomyelia after placement of lumbar subarachnoid fluid shunts, although neither condition was present before treatment. ${ }^{5}$ Intracranial MR imaging of a case of syringomyelia showed tonsillar herniation, flattening of the pons against the clivus, effacement of basal cisterns, bulging of the pituitary gland, and bowing of the chiasm. ${ }^{15}$

The relation between two pinholes in the dorsal thin dural sac and an arachnoid diverticulum at the origin of the root sleeve is unknown. An arachnoid diverticulum may result from CSF leakage from these two pinholes or these pinholes may be formed by mild body impact due to previously originated arachnoid diverticulum. There will be an opinion that these pinholes have resulted from previous EBP or surgical process. However, in the present case, CSF leakage from an arachnoid diverticulum was not found at all. Moreover the dura matter around these two pinholes had changed thinly only at this portion.

Our case is remarkable for considerable reduction of the syrinx in the cervicothoracic spinal cord on the 10th day after surgery. Placement of Gelfoam ${ }^{\mathbb{R}}$ around a dural tear brought about reduction of a syrinx in the cervical spinal cord 3 months after surgery in a patient with spontaneous intracranial hypotension. ${ }^{15)}$ The present case indicates that direct surgical suture of dural tears and placing Gelfoam ${ }^{\mathbb{R}}$ fixed with fibrin glue may reduce a syrinx effectively. Consequently, simple direct surgical repair of the dura mater should be considered to provide rapid cure of cerebellar tonsillar herniation acquired secondary to intracranial hypotension.

\section{Acknowledgments}

We are grateful to Tomoko Ota, M.D., Department of Cardiology, Brain Attack Center Ota Memorial Hospital, Fukuyama, Hiroshima for her echocardiographic examination of the patient prior to the operation.

\section{References}

1) Atkinson JLD, Weinshenker BG, Miller GM, Piepgras DG, Mokri B: Acquired Chiari malformation secondary to spontaneous spinal cerebrospinal fluid leakage and chronic intracranial hypotension syndrome in seven cases. J Neurosurg 88: 237-242, 1998

2) Berroir S, Loisen B, Ducros A, Boukobza M, Tzourio C, Valade D, Bousser MG: Early epidural blood patch in spontaneous intracranial hypotension. Neurology 63: 1950-1951, 2004

3) Christoforidis GA, Mehta BA, Landi JL, Czarnecki EJ, Piaskowski RA: Spontaneous intracranial hypotension: report of four cases and review of the literature. Neuroradiology 40: 636-643, 1998

4) Fishman RA, Dillon WP: Dural enhancement and cerebral displacement secondary to intracranial hypotension. Neurology 43: 609-611, 1993

5) Johnston I, Jacobson E, Besser M: The acquired Chiari malformation and syringomyelia following spinal CSF drainage: a study of incidence and management. Acta Neurochir (Wien) 140: 417-428, 1998

6) Moayeri NN, Henson JW, Schaefer PW, Zervas NT: 
Spinal dural enhancement on magnetic resonance imaging associated with spontaneous intracranial hypotension. Report of three cases and review of the literature. J Neurosurg 88: 912-918, 1998

7) Mokri B: Spontaneous cerebrospinal fluid leaks: from intracranial hypotension to cerebrospinal fluid hypovolemia - evolution of a concept. Mayo Clin Proc 74: 1113-1123, 1999

8) Mokri B, Parisi J, Scheithauer B, Piepgras DG, Miller GM: Meningeal biopsy in intracranial hypotension: meningeal enhancement on MRI. Neurology 45: 1801-1807, 1995

9) Pannullo SC, Reich JB, Krol G, Deck MD, Posner JB: MRI changes in intracranial hypotension. Neurology 43: 919-926, 1993

10) Pleasure SJ, Abosch A, Friedman J, Ko NU, Barbaro N, Dillon W, Fishman RA, Poncelet AN: Spontaneous intracranial hypotension resulting in stupor caused by diencephalic compression. Neurology 50: 1854-1857, 1998

11) Rabin BM, Roychowdhary S, Meyer JR, Cohen BA, LaPat KD, Russell EJ: Spontaneous intracranial hypotension: spinal MR findings. AJNR Am J Neuroradiol 19: 1034-1039, 1998

12) Rando TA, Fishman RA: Spontaneous intracranial hypotension: report of two cases and review of the literature. Neurology 42: 481-487, 1998

13) Renowden SA, Gregory R, Hyman N, Hilton-Jones D: Spontaneous intracranial hypotension. J Neurol Neurosurg Psychiatry 59: 511-515, 1995

14) Schievink WI, Tourje J: Intracranial hypotension without meningeal enhancement on magnetic resonance imaging. Case report. J Neurosurg 92: 475-477, 2000

15) Sharma P, Sharma A, Chacko AG: Syringomyelia in spontaneous intracranial hypotension. Case report. J Neurosurg 95: 905-908, 2001

16) Shievink WI, Meyer FB, Atkinson JLD, Mokri B: Spontaneous spinal cerebrospinal fluid leaks and intracranial hypotension. J Neurosurg 84: 598-605, 1996

Address reprint requests to: Tatsuya Ohtonari, M.D., Department of Spinal Surgery, Brain Attack Center Ota Memorial Hospital, 3-6-28 Okinogami-cho, Fukuyama, Hiroshima 720-0825, Japan.

e-mail: ohtonari@shouwa.or.jp 